\title{
A Remote Social Robot to Motivate and Support Diabetic Children in Keeping a Diary
}

\author{
Esther J.G. van der Drift, \\ Robbert-Jan Beun \\ Utrecht University \\ P.O. Box 80.089 \\ 3508 TB Utrecht \\ evddrift@gmail.com, \\ r.j.beun@uu.nl
}

\author{
Rosemarijn Looije, Oliver A. \\ Blanson Henkemans \\ TNO \\ P.O. Box 23 \\ 3769 ZG Soesterberg \\ rosemarijn.looije, \\ olivier.blansonhenkemans@tno
}

\author{
Mark A. Neerincx \\ Delft University of Technology \\ Faculty of EEMCS \\ Mekelweg 4 \\ 2628 CD Delft \\ m.a.neerincx@tudelft.nl
}

.nl

\begin{abstract}
Children with diabetes can benefit from keeping a diary, but seldom keep one. Within the European ALIZ-E project a robot companion is being developed that, among other things, will be able to support and motivate diabetic children to keep a diary. This paper discusses the study of a robot supporting the use of an online diary. Diabetic children kept an online diary for two weeks, both with and without remote support from the robot via webcam. The effect of the robot was studied on children's use of the diary and their relationship with the robot. Results show that children shared significantly more personal experiences in their diaries when they were interacting with the robot. Furthermore, they greatly enjoyed working with the robot and came to see it as a helpful and supportive friend.
\end{abstract}

\section{Categories and Subject Descriptors}

H.1.2 [User/Machine Systems]: Human factors; I.2.9 [Robotics]: Operator Interfaces; J.4 [Computer Applications]: Social and Behavioral Sciences.

\section{General Terms}

Design, Experimentation, Human Factors, Verification.

\section{Keywords}

Social robot; remote robot; diabetes; diary; embodiment; adherence; compliance; bonding; engagement; relationship; childrobot interaction; human-robot interaction.

\section{INTRODUCTION}

It is estimated that worldwide more than 490 thousand children between the ages of 0-14 suffer from diabetes mellitus type 1, and this number continues to rise explosively [1]. Diabetes is a chronic illness that impacts a child's life in almost every aspect. Effective self-management of diabetes is complex and involves many varied activities related to dosing insulin, monitoring metabolic control and regulating diet and exercise to name just a few. This can be especially challenging for children that are diagnosed at a very young age. They frequently have trouble

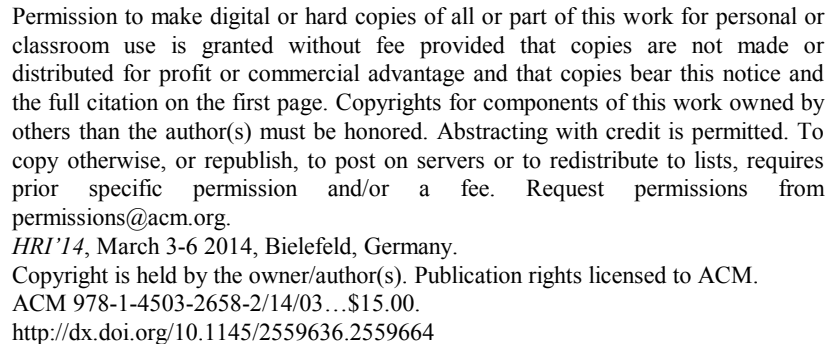

Permission to make digital or hard copies of all or part of this work for personal or classroom use is granted without fee provided that copies are not made or distributed for profit or commercial advantage and that copies bear this notice and the full citation on the first page. Copyrights for components of this work owned by others than the author(s) must be honored. Abstracting with credit is permitted. To copy otherwise, or republish, to post on servers or to redistribute to lists, requires prior specific permission and/or a fee. Request permissions from permissions@acm.org.

HRI'14, March 3-6 2014, Bielefeld, Germany.

Copyright is held by the owner/author(s). Publication rights licensed to ACM.

ACM 978-1-4503-2658-2/14/03 ..\$15.00.

http://dx.doi.org/10.1145/2559636.2559664

coping with their diabetes [2], and are at risk for developing depression, anxiety disorders or eating disorders [3, 4, 5]. Health care providers advise children to keep a diary to monitor their health and how they feel on a daily basis so that appropriate treatment adjustments can be made [6]. Keeping a diary can significantly contribute to the quality of life of children with diabetes. A diary provides insight into patterns between blood glucose values and daily activities so that the child can better manage his/her diabetes. But despite this advantage of keeping a diary, children rarely take the time to do it or they do not see the value of keeping a diary. Digital diaries have been found to yield better compliance and accuracy in diary recording compared to paper diaries [7], but the problem of motivating children to start using these diaries still exists.

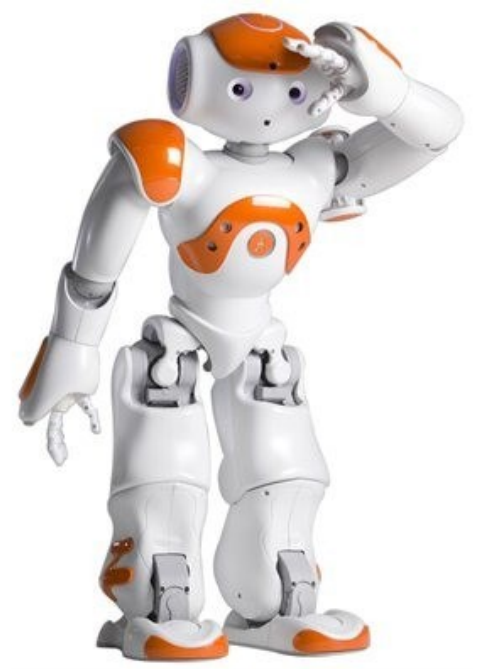

Figure 1. NAO robot by Aldebaran Robotics

The EU-funded ALIZ-E project www.aliz-e.org aims to develop a social robot to support chronically ill children in their selfmanagement. The scenario in which the robot is being tested is based on a medical setting where children recently diagnosed with diabetes spend one or two weeks in the hospital. During this time, the child and its parents are intensively trained and educated to manage the child's diabetes. But of course self-management of diabetes does not end there. It is an active and lifelong process that involves shifting and sharing responsibility for care tasks and decision-making between parents and child. The ALIZ-E project focuses on the potential role of a robot (Figure 1) in this process as an educator, motivator and companion that remains interesting to the child on the long term [8]. 
This paper describes an experiment which aims to improve children's diary adherence by means of a social robot. We created a scenario in which diabetic children keep a diary from home for two weeks, intermittently interacting with the robot via videoconferencing software. We studied the effect of the robot on the children's diary adherence, their engagement in the activity and their relationship with the robot.

\section{RELATED WORK}

Social robots are increasingly designed to be our pets, assistants, teachers and even emotional companions [9, 10]. They can positively affect people's motivation and compliance in areas such as education, health and well-being [11, 12]. The effectiveness of a social robot as a motivator largely depends on its ability to persuade its user. Persuasion is defined as "an attempt to shape, reinforce, or change behaviors, feelings, or thoughts about an issue, object or action" [13]. In this study, the issue we are addressing is the low diary adherence of children with diabetes. The robot attempts to persuade the child to keep a diary by using a variety of means that have been proven to be effective in humanrobot interactions. Directly coercing the child into better keeping a diary is not an option for two reasons: first, coercion often has the opposite effect and causes the child to rebel even more [14]; and second, from a practical standpoint it is impossible to uphold this approach because the parents will not always be around to check up on their child. Rather than making active attempts to change the child's attitude and behavior, the focus should be on positively reinforcing the child's actions and utilizing the potential bond between the robot and the child as an incentive for the child to keep a diary.

To cultivate long-term relationships with users, the robot needs to engage in social behavior and dialogue [15]. Social dialogue includes things like greetings, chatting about general topics like the weather, and exchanging personal preferences [16]. Selfdisclosure and empathy are known to greatly contribute to the closeness between conversational participants [17, 18, 19]. This effect is stronger with a physically embodied robot than with a virtual agent. There are many other factors we could consider which have the potential to positively influence children's diary adherence and long-term relationship with the robot. However, we feel that a robot which exhibits self-disclosure, empathy and physical embodiment provides a good starting point for this formative evaluation. These aspects can easily be implemented into the robot's dialogue and behavior.

\subsection{Self-disclosure}

Studies have shown that self-disclosure plays a central role in the development and maintenance of relationships as well as psychological well-being $[17,20,21,22]$. Self-disclosure is defined as "sharing information with others that they would not normally know or discover" [23]. Once a person engages in selfdisclosure, it is implicitly expected that the other conversational partner will also disclose information (norm of reciprocity). Selfdisclosure has been linked to a person's likeability [20]. People who engage in intimate disclosures tend to be liked and trusted more than people who disclose at lower levels. People tend to also like robots better when they disclose affective rather than taskrelated information in collaborative tasks [24]. Mutual affective self-disclosure between the child and the robot can contribute to the depth and quality of their interaction, and ultimately their relationship. Self-disclosure also contributes to the diabetes selfmanagement of the child. Sharing daily experiences in the diary and reflecting on this information can help the child gain insight into how they can better manage or cope with their diabetes and ultimately improve their quality of life. In order to encourage the child to disclose information, we propose to utilize the reciprocal nature of self-disclosure and have the robot frequently disclose information about itself in order to encourage the child to do the same.

\subsection{Empathy}

Empathy plays a key role in patient-centered therapy, because it implies the apprehension of another's inner world and a joint understanding of emotions [25]. One of the most comprehensive definitions of empathy is by Davis [26], who defined it as "the capacity to take the role of the other, to adopt alternative perspectives vis-à-vis oneself and to understand the other's emotional reactions in consort with the context to the point of executing bodily movements resembling the other's". Robots cannot feel empathy, but they can emulate it in their behavior, for example by:

- Showing empathic concern for others;

- $\quad$ Taking the perspective of another;

- Emotionally identifying with fantasy characters in books, films, etc.;

- Expressing negative feelings in response to the distress of others.

We propose to incorporate empathy in the robot's behavior by showing sympathy or concern when the child says to be feeling down, and by reacting positively when the child is in good spirits. The robot also expresses its concern in asking the child how he or she handles certain issues related to diabetes (i.e. fear of exercising or pricking insulin in public).

\subsection{Embodiment}

Social robots do not necessarily need a physical body to interact with their users. Their tasks can often be performed just as well by a virtual $3 \mathrm{D}$ representation or avatar of the same robot, which costs considerably less and is much more robust. But having a physical form does offer substantial benefits compared to virtual robots. Embodied robots (robots with a physical presence) are more appealing and perceptive of the world around them than non-embodied robots [27]. Participants' impression of the robot's watchfulness, helpfulness, and enjoyableness is significantly affected by embodiment. In a study on a socially assistive robot exercise coach for the elderly [28], participants strongly preferred a physically embodied assistive robot over the virtually simulated one. The interaction with the physical robot was rated as more enjoyable and useful. Physical embodiment has also been found to evoke a higher degree of user engagement and presence [29].

The positive effects of having a physical body were shown to still be prevalent when the robot is shown remotely via a camera. The social presence of a remote physical robot was almost the same as a robot that was located physically near the user [30]. A remote projected robot and a physically present robot were found to be equally engaging and elicited equal disclosure from the user.

The ALIZ-E project develops a robot for long-term interaction with chronically ill children undergoing treatment. It is important that the children interact with the same robot throughout this entire period to provide a consistent experience. They interact first with the physical robot in the hospital, and later continue with the same robot shown remotely from home. 


\subsection{Hypotheses}

The bond between the robot and the child can serve as an incentive for the child to keep a diary. To this end, the robot engages in self-disclosing behavior and encourages the child to do the same. It also shows emulated empathy and concern for the child. And because people generally find interaction with a (remote) physical robot the most rewarding experience, we choose to study the effect of the robot on the child's self-disclosure in the diary, engagement in the activity, and bonding with the robot. This leads us to the following hypotheses:

1. Adherence: the robot encourages the child to self-disclose more information in their diaries.

2. Engagement: the robot has a positive effect on the child's engagement in the activity.

3. Bonding: the robot conveys a sense of trust and understanding of the child.

In order to test these hypotheses an experiment in a real-world setting was conducted.

\section{IMPLEMENTATION}

\subsection{Robot}

The NAO robot (developed by Aldebaran Robotics) was used in this experiment. NAO is well-suited for interaction with children largely due to its friendly childlike appearance. Although its face lacks the capability to display emotions, the robot is able to show a wide array of emotions through its body language. The expressions used in this experiment were idle behavior and emotional expressions that were pretested in [31]. The authors found that the recognition rates for these basic emotions (e.g. happiness, sadness and surprise) were relatively high, between $68 \%$ and $99 \%$ accuracy. Idle behavior consisted of small body movements such as moving the head and hand positions while speaking or waiting for the user to answer. The robot in the experiment was given the unisex name "Charlie" in order to appeal to both boys and girls. A background story was written for the robot to answer basic questions about its likes and preferences, and about its reason for participating in the experiment. In the story Charlie is a hospital care robot in training and it hopes the child can help it learn more about diabetes by keeping a diary together. When the robot engages in selfdisclosure, it does so keeping this background story in mind. The robot has an inquisitive character, and regularly asks the child about what it is like having (and coping with) diabetes.

\subsection{Dialogue model}

A dialogue model was developed that structured the robot dialogue in an orderly fashion. The model consisted of two parts: the diary-related dialogue, and the interpersonal dialogue.

1. Diary-related dialogue: any dialogue related to the task of keeping a diary. This includes the login process, explaining of diary sections and filling in the diary itself. This part of the dialogue was very structured, and did not allow for much flexibility other than the child choosing what section he/she wanted to start with. Essentially this part of the dialogue was the same for every child.

2. Interpersonal dialogue (or 'small talk'): any dialogue not directly related to the task. There were two different types of small talk.
- Self-disclosure small talk was used for the robot to share information about itself and optionally ask the child to do the same. For example, the robot told the child about its favorite pets, and then asked the child if he/she has any pets.

- Diabetes-related small talk was used for the robot to talk about what it learned in school about diabetes (e.g. doing sports when having diabetes) and ask the child some basic questions about this topic (e.g. "How do you handle your diabetes when you do sports?"), and if he/she has any fears related to his/her diabetes (e.g. fear to prick blood glucose, or fear to exercise).

The small talk was used to enrich the dialogue between the robot and the child, with the goal of creating a bond between them.

\subsection{Wizard of $\mathrm{Oz}$}

The main deliverable of the ALIZ-E project is an integrated and autonomous system comprising different modules. But because this system is developed incrementally and not all features have fully matured, some of the robot's functionality is simulated. The participants interacted with the robot which they believed to be autonomous, but which was actually controlled by the experiment leader in a Wizard of $\mathrm{Oz}(\mathrm{WoOz})$ setup. The NAO robot was capable of performing some actions autonomously, such as movements while speaking, 'blinking' of the eyes by switching LEDs on and off, and speaking (text-to-speech). Other actions could not be performed autonomously and needed human intervention. The experiment leader interpreted the user input (speech, gestures, and actions) to respond to the user by choosing the relevant remarks from the dialogue model. Certain movements such as cheering or nodding were initiated by the experiment leader by clicking the corresponding button in the $\mathrm{WoOz}$ interface. Updating the user model was also done by hand when the user provided new information. The dialogue was scripted in the dialogue model. In rare incidents, the experiment leader could type a response and have the robot say it via the text-to-speech module. The timing of interpersonal dialogue was largely up to the experiment leader. She could choose from one of the prescripted small talk phrases whenever a related topic was mentioned, or when there was a moment of silence.

\subsection{Diary}

The study required a diary that allowed the children to not only record their measured values, but that also had room for them to express how they feel. This allows the robot to respond empathetically to the emotional content of the diary. To this end, we chose to adapt an online diary for use in our experiment. Mijn Zorgpagina (literally 'my care page') is an initiative from Diabetesvereniging Nederland (DVN), the Dutch Association for Diabetes. In cooperation with DVN there were extra sections added to the diary to record the child's emotional well-being. The diary consisted of three different sections (see Figure 2): the 'values' section which consisted of different 'lines' for the recording of blood glucose values, carbohydrate intake, insulin doses and exercise; the 'emotions' section in which the child could rate his/her appetite, energy and mood levels using three sliders; and finally the 'daily activities' section which was a text box in which the children could write anything they wanted to share about their day. 


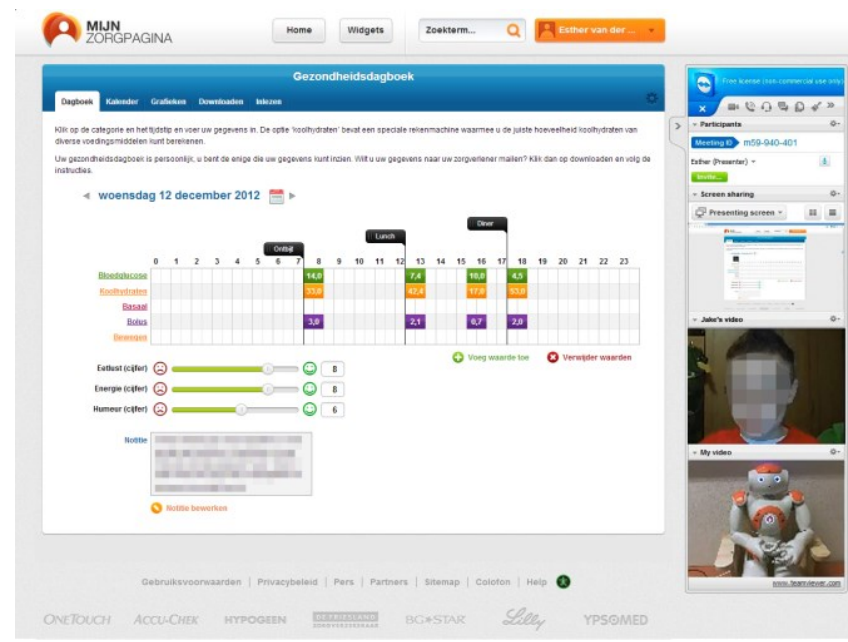

Figure 2. Screen as seen by the child showing diary and video conferencing software

In Figure 2, the video conferencing software (TeamViewer) we used is shown on the right-hand side of the screenshot (face of the child is blurred for privacy reasons). We used TeamViewer to enable desktop-sharing and communication via webcam and audio. Although the children could $\log$ into the diary from anywhere, we chose to provide each of them with a laptop that was stripped down to the bare minimum. The main reason for this was that we wanted to make sure all diaries were accessed from the same platform so that there would be no technical difficulties on their end. The children's accounts were set up in a way so that they would automatically $\log$ in when there was an internet connection. The child could then invite Charlie for a session. The sessions were all scheduled in advance. If they encountered any issues while attempting to $\log$ in or connecting to Charlie, the children could refer back to the child-friendly user manual that we provided or contact the experiment leader.

\section{METHODS}

\subsection{Participants}

Six children affiliated with Dutch hospital Rivierenland in Tiel participated in the formative evaluation. They were recruited with the help of a diabetes nurse who informed the children and their parents of the experiment. Interested parents were contacted and they received further information. They could then decide whether they wanted their child to participate. The group consisted of two girls and four boys, aged 9-12 $(\mathrm{M}=10.8, \mathrm{SD}=1.3)$. All children were diagnosed with diabetes mellitus type 1 . On average, they had diabetes for six years. None of the children had any prior experience in keeping a digital diary.

\subsection{Experiment design and procedure}

One week before the start of the experiment, we visited each of the children individually at home to introduce them to the robot. We explained the goal of the research and there was an opportunity to ask questions. The robot then introduced itself and asked the child to do the same. After that, we scheduled the sessions with the robot for the following two weeks. Children who forgot their appointments with Charlie were called to remind them. We feared that without reminders, children would forget their study participation, at the cost of valuable data. The goal of this experiment was gathering knowledge on the target group and their use of an online diary with or without the robot. As such the experiment can be seen as a formative evaluation. The number of participants was small and there was only limited time available for interaction with the robot. Therefore we decided to do a within-subjects design; this entails that all children kept a diary both with and without the robot. They did this for a total of 11 days. This allowed us to clearly see the differences in diary use for the same children in both conditions. We made two groups of three participants to spread the workload. This means that half of the children started on day 1 and finished on day 11, whereas the other half started on day 2 and finished on day 12. The sessions with and without the robot alternated every day. This excluded the weekends, which were always without the robot because access to the facility where the robot was stored was not possible. The children participated while in the comfort of their own homes using video conferencing software to contact the robot.

\subsection{Measures}

Below we present the metrics used for each of the three hypotheses.

1. Adherence: In this context adherence is defined as the extent to which children keep an accurate account of their values, mood and activities in their diaries on time (meaning on the same day the values were measured). If the robot has a positive effect on diary adherence, we expect to see 1) more completed diary entries with the robot, and 2) richer diary content (experience sharing/self-disclosure). We determine whether this is true by logging children's use of the diary and comparing the contents of the diary between sessions with and without support from the robot. Furthermore, we measure likeability and trust, because literature found these to be contributing factors for self-disclosure.

2. Engagement: An engaged child typically spends more time and is focused on the activity, and takes an active role in the activity. Comparing the time spent on the diaries between sessions with and without the robot can tell us what the influence of the robot on engagement is. Active participation and attention are measured by the experiment leader by observing the child's interaction with the robot via the webcam. A child is thought to take an active role in the conversation whenever he/she asks or tells the robot something of his/her own accord instead of waiting for the robot to ask something first. We can determine attention by observing the child's gaze direction. When the child looks away from the diary and the robot, he/she is distracted.

3. Bonding: A bond between two conversational partners is characterized by emotions such as affection and trust. To investigate to what extent interaction with the robot created a bond, we used a post-condition questionnaire which focused on the degree of relatedness the children experienced with the robot. When a child trusts the robot, believes it has feelings, and thinks that it can truly understand him/her, the feeling of relatedness is strong.

\subsection{Instruments}

The children were asked to complete a total of 7 questionnaires. The questions were phrased in simple terms and emoticons were added to Likert-scale questions for clarification (cf. [32]). The pre-condition questionnaire was answered prior to the experiment, and inquired about demographic information, interests and expectations. We used this questionnaire to learn more about our participants and whether they understood the experiment 
correctly. After each interaction session $(5 x)$ with the robot, the children were again asked to fill in a questionnaire. This questionnaire only contained two questions which inquired about the children's ratings of the robot and the diary. This data can be used to determine whether there are any changes over time. Finally, the children answered a post-condition questionnaire after all the sessions were completed. In this questionnaire we asked them about their overall judgment of the robot and the diary, the degree of relatedness with the robot, and feelings towards the robot. These data were mostly used to determine the extent of the relationship between the child and the robot.

Furthermore, we also made use of three different types of logs. The diary logs were provided by MijnZorgpagina and provided insight into the children's use of the diary on days when the robot was not present. The $\mathrm{WoOz}$ logs were automatically generated whenever a command was sent to the robot. We used these to determine use of the diary with the robot. Lastly, the experimenter leader's observation logs were used to note any changes in experience sharing, active participation and attention of the children during the sessions. Miscellaneous observations were also noted in these logs.

Initially we had planned to record all interaction sessions on video to carefully note any changes in active participation and attention. However, due to a technical problem, the video conferencing software crashed when the recording software was started. We came to rely on the experiment leader's observations for data regarding the children's engagement.

\subsection{Setup}

Figure 3 provides a schematic overview of the experimental setup. To mimic the natural sitting position of humans during video calls, the robot (1) was seated in a chair during the interaction sessions. The webcam (2) was aimed at the robot from an angle slightly higher than eye level because we wanted the children to believe it was operating its own laptop. During the interaction sessions, the experiment leader (3) was seated out of view. She was in control of the robot's actions using a laptop and an extra screen (4). She was also able to see the screen and webcam of the child using the video conferencing software (5) on a second laptop. The experiment leader made as little noise as possible so as not to alert the child to her presence. To this end, we used a silent mouse (6) that lacked the "click" sound when a button was pressed.

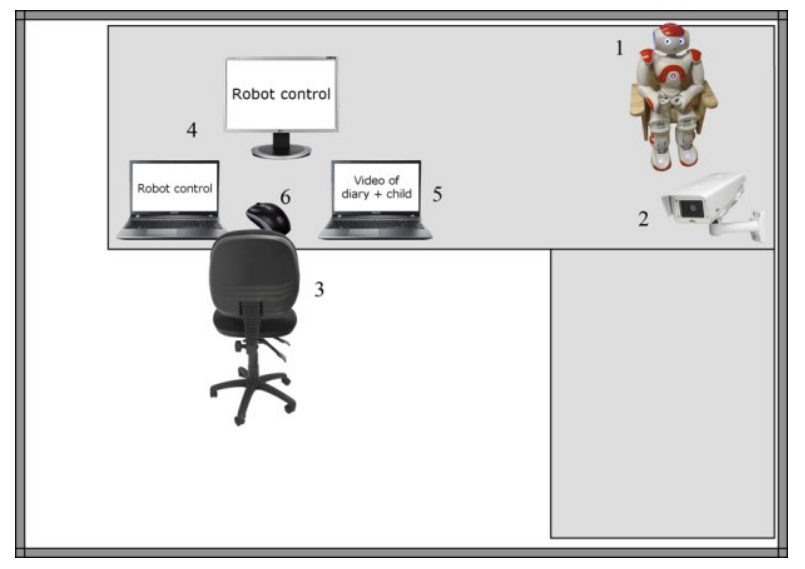

Figure 3. Experimental setup

\section{RESULTS}

All six children completed the full experiment and the associated questionnaires. Diary use prior to the experiment was found to be very low; none of the children kept a diary on a daily basis. Five out of six children used an insulin pump and three of them indicated they did not keep a diabetes diary (anymore) because they were able to read out values from their pumps if needed. Only one child said that he currently kept a diary all by himself, but after enquiring about his diary use he admitted to only doing it once every three months when it was required for a hospital consultation. All of the children said to be at least moderately interested in technological advances such as robots or gadgets.

\subsection{Adherence}

The log data from the diary revealed that not all children completed their diaries on time (meaning on the same day the values were measured). When a diary entry was not completed on time, it was usually completed the day after. When they were not interacting with the robot, two of the children sometimes neglected to keep their diary. One of them missed all (6) entries, the other left only 2 entries uncompleted. Three children logged on multiple times (1,2 and 8 times) to supplement values as new information became available. The differences between the conditions in filling out the diary were not significant due to the high interpersonal variation. Figure 4 shows the differences in diary completion between conditions in percentages of the total actions performed. For example, all children fully completed the diary in the robot condition, but some of them also supplemented the diary with extra information when it became available in this condition. The total of all these actions adds up to 100 percent.

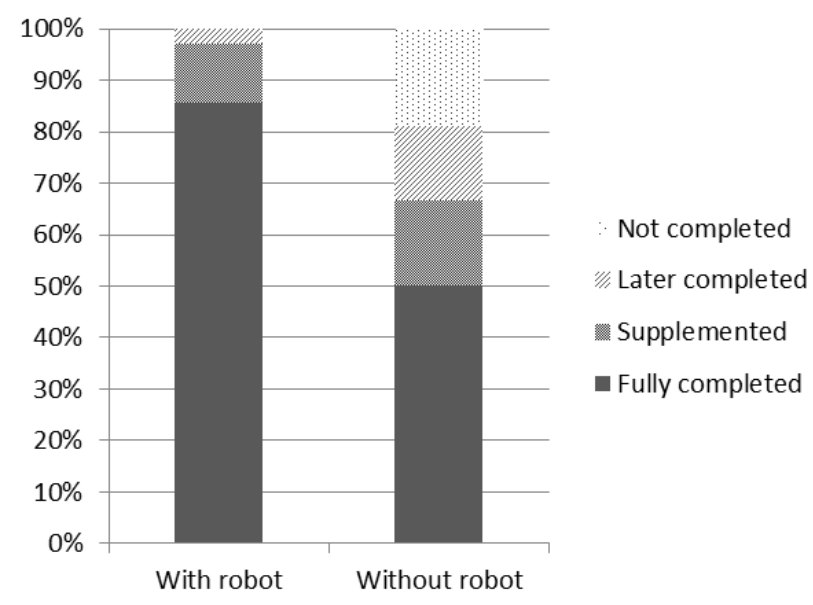

Figure 4. Diary actions performed with and without robot

We compared the amount of characters used in the daily activity logs between the days with the robot and without the robot as an indicator for experience sharing. It was found that children wrote significantly more in their logs when they were interacting with the robot $(\mathrm{M}=83.10, \mathrm{SD}=43.96)$ than without the robot $(\mathrm{M}=36.8$, $\mathrm{SD}=54.31) ; \mathrm{t}(5)=4.13, \mathrm{p}=.009$; but there were clear differences between participants. In table 1 it is evident that one participants (5) always filled in high quantities of data, whereas some others filled in less on days without the robot (2 and 3), or nothing at all when the robot was absent (1 and 4).

Overall, the children rated the diary positive throughout the experiment. The diary received an average rating of 3.9 on a scale from 1 to 5 . 
Table 1 Average amount of characters in daily activities logs

\begin{tabular}{|l|l|l|}
\hline Participant & With robot & Without robot \\
\hline 1 & 60.4 & 0 \\
\hline 2 & 36.6 & 15.7 \\
\hline 3 & 86.4 & 13.5 \\
\hline 4 & 78.8 & 0 \\
\hline 5 & 165.6 & 141 \\
\hline 6 & 70.8 & 50.8 \\
\hline \hline Mean & $\mathbf{8 3}(\mathbf{S D}=\mathbf{4 0})$ & $\mathbf{3 7}(\mathbf{S D}=\mathbf{5 0})$ \\
\hline
\end{tabular}

\subsection{Engagement}

We were unable to validate whether there was a difference in the time spent on the diary due to a gap in the log data. The time spent on the daily activities log was not recorded on the days without the robot, so the data from both conditions were unequal. However, we did see how the time spent on the diary changed over time. The first session with the robot typically took between 10 to 15 minutes, as did the second session at home without the robot. In later sessions, the time taken dropped and stabilized around 5-6 minutes for both conditions. This was due to the fact that by then the children knew their way around the diary and became more efficient in completing their task.

The younger children (aged 9) $(\mathrm{N}=2)$ were much more open and talkative than the older children (11-12) $(\mathrm{N}=4)$. Their answers were longer and their sessions typically took 1-2 minutes longer than those with the older children. They were more likely to ask the robot about its personal life than the other children were. Older children appeared to be less interested in the robot's life and did not ask it as many questions. They usually answered the robot with a simple "yes" or "no". There was no discernible difference between genders in interaction styles with the robot.

The children were very patient when they had to wait a while for Charlie to answer. This was apparent in the way that the children remained still and focused even when the robot did not do anything for a while. Overall the children were focused. They did not allow themselves to be distracted by background noises or siblings and focused solely on the diary and to a slightly lesser extent on the robot. When the robot spoke, their attention visibly shifted to the robot for a short while, but then quickly back to the diary. This was apparent from their gaze direction which was visible on webcam.

\subsection{Bonding}

In the post-condition questionnaire, the children were asked to agree or disagree with a number of statements on a scale of 1 to 5 , where 1 meant 'completely disagree' and 5 meant 'completely agree'. Figure 5 summarizes the results from the post-condition questionnaire.

The robot received high ratings on all questions. Especially high (average rating above 4) was the rating of the robot's trustworthiness and its human-like behavior. Furthermore, when we asked the children what they liked about Charlie, they said they liked the fact that it asked questions about the things they did that day, as well as share with them his own daily activities.
Figure 5 Post-condition questionnaire ratings $(\mathrm{N}=6)$

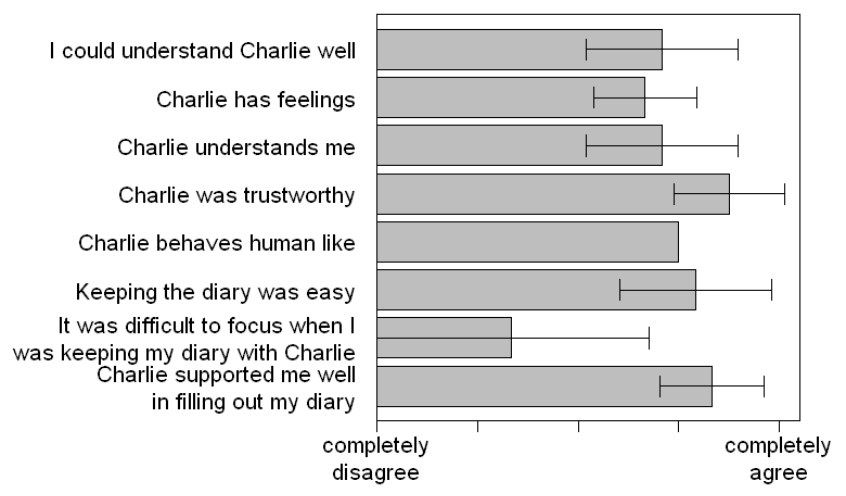

We also asked the children how they viewed the robot. Five out of six children chose the option 'Friend'. One child said that Charlie was more like a peer, and another said that it was a device/robot to him (but also a friend). The children rated the robot after every session. On average, the robot received an average rating of 4.2 on a scale of 1 (lowest) through 5 (highest).

Furthermore, we noticed the children often smiled or laughed at the things the robot said, and they waved back to the robot when it greeted them. Overall they were very friendly towards it. The most positive reactions were elicited when the robot immediately responded to the ratings for the emotions and daily activities. The children seemed to think that it really understood them. The level of detail in the questions some of the children asked was high, regardless of their age. For example, when Charlie said that it had played soccer with friends, they would ask it about the final score and who the goal keeper was. One child even asked about Charlie's birthday, and when it said that was February 2nd, she wished it a happy birthday in advance, much like humans would do to each other.

\section{DISCUSSION}

\subsection{Self-disclosure and diary use}

Children completed their online diary more often when they were keeping it with the robot than when they were keeping it alone. This was to be expected, as the children were actively notified by the experiment leader on the days they were scheduled to fill in the diary with the robot. However, after the children had interacted with the robot, they also logged in the diary later that day to supplement the diary, on their own initiative. This suggests that the robot was motivational by itself. A critical note with regard to the diary completion is that we called the children who forgot their appointments with Charlie. Considering the small sample size, we felt it was more important to have as many interaction sessions as possible to learn how the robot's selfdisclosure influenced diary adherence. Moreover, children consistently filled in more information in their daily activities logs (the bottom part of the diary) when they were keeping their diary with the robot, than when they were keeping it by themselves. Thus, regardless of whether or not the children were reminded of their appointments, when they did interact with the robot, they filled in much more information. The robot's self-disclosure appeared to be a strong motivator for the child to disclose information in return. We can confirm the hypothesis that the robot encourages the child to share more information in his/her diary. 


\subsection{Embodiment and children's engagement}

From the log data we gathered that the time taken to fill in the diary dramatically decreased after the first two sessions. This can be explained by the fact that during the first session the children listened to the robot explaining every diary section, while in subsequent interactions this explanation was optionally accessible (and rarely asked for). Due to the failed recordings of the sessions, the data on children's interaction behavior was elicited from notes made by the experiment leader during the study. These notes were taken systematically and helped explain some of the results, but unfortunately they could not be statistically validated. The observations showed a strong difference in active participation between the younger children, who were very open and talkative, and the older children who were less open and talkative. Possibly, the background story for Charlie was not convincing or appealing enough for the older participants in this study. This is in line with the commentary we received from an older child (13) during the pilot study we performed prior to the experiment ("I think the story is too childish for 11-12 year olds"). Overall, the experiment leader had the impression that the children paid close attention to the robot and the diary. They did not allow themselves to be distracted by background noises, siblings or parents. While some parents chose to supervise their child during the sessions, they did not appear to be a distracting factor. We did however see some mixed results in the answers on the question whether it was difficult to focus when they were keeping a diary with Charlie (in the post-condition questionnaire). But this might also be due to the slow internet connections of some children. Occasionally there were very large delays in the sound transmission, which made it difficult to understand what Charlie was saying. Since we did not have sufficient data on the children's engagement with the diary without the robot present, it is difficult to say whether the robot had a positive effect on the child's engagement in the activity. Therefore we cannot confirm the second hypothesis.

\subsection{Robot's understanding and trust}

Overall, the robot was received very well by the children. They greatly enjoyed interacting with Charlie. They believed it had feelings, was able to understand them, and that it behaved humanlike, which are signs of empathy. However, we have to be careful in saying the conveyed sense of trust and understanding of the child was because of the robot (hypothesis 3), because occasionally part of the dialogue was typed by the experiment leader directly and converted to speech by the robot when there was no suitable answer in the dialogue model. Although most of the dialogue used was in fact pre-scripted, the comments that were made on the fly could have still somewhat skewed our results.

\subsection{Future work}

Future work in the area of robot-driven diary support could benefit from sentiment analysis, which refers to the use of natural language processing, text analysis and computational linguistics to identify and extract subjective information from source materials (i.e. the diary). Sentiment analysis would allow the robot to more accurately predict and interpret the emotional state of the child and choose the appropriate response. In our experiment, a human actor had to interpret the data and 'translate' this for the robot. Ultimately the goal of the ALIZ-E project is an integrated system which operates without human intervention. There still needs to be done a significant amount of work in the area of speech recognition and interpretation to achieve this goal.
This paper presented a first experiment in which we succeeded to include six children with diabetes, who interacted with the application over an extended period of time. Due to the small number of participants, a within-subjects design was applied. Based on the results, we will be able to get other hospitals interested and conduct a between-subjects experiment with more participants. Participation of diabetic children remains crucial, because children without diabetes do not have the intrinsic motivation or ability to fully complete a diabetes diary.

In the current experiment there was no explicit feedback about the relationship between blood glucose, mood and daily activities. In future work it would be interesting to add this type of feedback to the dialogue and to discover patterns together with the robot. We see opportunities for two follow-up experiments: (1) comparing children who keep a diary either with or without the robot, and (2) comparing their diary usage for a robot which does or does not exhibit self-disclosure, empathy and embodiment. Last but not least, the bonding effects of co-located and remote interactions should be tested: How the bonding transfers or evolves from colocated experiences with the robot in the hospital to remote interactions via webcam, and vice versa.

\section{CONCLUSION}

This paper investigated the contribution of a social robot to keeping an online diary together with diabetic children. A social robot can enhance the pleasure of the activity, and therefore the motivation of the child. Especially once the robot and the child really get to know each other, the child starts to consider the robot as a friend and he/she really opens up to it. Keeping an online diary together with a social robot can contribute to a better diary adherence. The robot utilizes aspects of physical embodiment, self-disclosure and empathy in its behavior and dialogue to achieve this goal. When we take into account that the diary use prior to this study was almost non-existent, this is a considerable improvement. Keeping a record of the values, emotional wellbeing and the daily activities allows the child to make meaningful inferences about the relationship between these three variables. This positively influences the child's self-reflection capabilities, which in turn contributes to his/her self-management. The robot could help the children overcome the initial hurdles of taking charge of their own diabetes self-management. The addition of a robot does not have to be detrimental to the children's feeling of independence, which becomes increasingly important as they reach puberty. This study provides a sound foundation for future research into robot characteristics and their effect on emotional support for chronically ill children.

\section{ACKNOWLEDGMENTS}

This work is funded by the EU FP7 ALIZ-E project (grant 248116). We would like to thank the diabetic children and medical staff from the ZRT hospital in Tiel (the Netherlands) and Diabetesvereniging Nederland (DVN) for their cooperation.

\section{REFERENCES}

[1] International Diabetes Federation. 2012. IDF diabetes atlas. Retrieved August 13, 2013 from http://www.idf.org/diabetesatlas/5e/diabetes-in-the-young

[2] Whittemore, R., Kanner, S. and Grey, M. 2004. The influence of family on psychological and psychosocial health in youth with type 1 diabetes: a systematic review. In Melnyk, B.M. \& Fineout-Overholt. Evidence-based practice 
in nursing and healthcare: a guide to best practice, Williams \& Wilkins, Philadelphia, CD22-73-CD22-87.

[3] Grey, M., Whittemore, R. and Tamborlane, W. 2002. Depression in type 1 diabetes in children: natural history and correlates. Journal of Psychosomatic Research, 53, 4, 907 11 .

[4] Kovacs, M., Goldston, D., Obrosky, D.S. and Bonar, L.K. 1997. Psychiatric disorders in youths with IDDM: rates and risk factors. Diabetes Care, 20, 36-44.

[5] Affenito, S.G. and Adams, C.H. 2001. Are eating disorders more prevalent in females with type 1 diabetes mellitus when the impact of insulin omission is considered? Nutrition Reviews, 59, 179-182.

[6] Rosenthal, S.M. 2008. The Canadian Type 2 Diabetes Sourcebook. John Wiley \& Sons Inc, Hoboken (New Jersey), 72-73.

[7] Palermo, T.M., Valenzuela, D. And Stork, P.P. 2004. A randomized trial of electronic versus paper pain diaries in children: impact on compliance, accuracy, and acceptability. Pain, 107, 3, 213-219.

[8] ALIZ-E. 2013. ALIZ-E research. Retrieved September 6, 2013 from http://www.alize.org

[9] Lee, K.M., Peng, W., Jin, S. and Yan, C. 2006. Can Robots Manifest Personality? An empirical test of personality recognition, social responses, and social presence in humanrobot interaction. Journal of communication, 56, 4, 754-772.

[10] Okita, S.Y. 2013. Self-Other's Perspective Taking: The Use of Therapeutic Robot Companions as Social Agents for Reducing Pain and Anxiety in Pediatric Patients. Cyberpsychology, Behavior, and Social Networking, 16, 6, 436-441.

[11] Chidambaram, V., Chiang, Y.H. and Mutlu, B. 2012. Designing persuasive robots: how robots might persuade people using vocal and nonverbal cues. In Proc. HRI 2012, ACM Press (2012), 293-300.

[12] Blanson Henkemans, O.A., van der Boog, P.J.M., Lindenberg, J., van der Mast, C.A.P.G., Neerincx, M.A. and Zwetsloot-Schonk, B.J.H.M. 2009. An online lifestyle diary with a persuasive computer assistant providing feedback on self-management. Technology and Health Care, 17, 3, 253267.

[13] Fogg, B.J. 2003. Persuasive technology: using computers to change what we think and do. Morgan Kaufmann Publishers, Amsterdam.

[14] Lask, B. 2003. Motivating children and adolescents to improve adherence. The Journal of Pediatrics, 143, 4, 430433.

[15] Baxter, P., Belpaeme, T., Cañamero, L., Cosi, P., Demiris, Y. and Enescu, V. 2011. Long-Term Human-Robot Interaction with Young Users. In Proceedings of HRI Workshop 2011, Lausanne.

[16] Higashinaka, R., Dohsaka, K. and Isozaki, H. 2008. Effects of self-disclosure and empathy in human-computer dialogue. In IEEE Spoken Language Technology Workshop 2008, Goa, India.
[17] Altman, I. and Taylor, D. 1973. Social penetration: The development of interpersonal relationships. Holt, Rinehart \& Winston, New York.

[18] Reis, H.T. and Shaver, P. 1988. Intimacy as an interpersonal process. In Duck, S. Handbook of personal relationships, Wiley, Chichester, England, 367-398.

[19] Moon, Y. 2000, Intimate exchanges: using computers to elicit self-disclosure from consumers. The Journal of Consumer Research, 26, 4, 323-339.

[20] Collins, N.L. and Miller, L.C. 1994. Self-Disclosure and Liking: A Meta-Analytic Review. Psychological Bulletin, $116,3,457-475$.

[21] Jourard, S.M. 1964. The transparent self. Van Nostrand, Princeton.

[22] Laurenceau, J.P., Feldman Barrett, L. and Pietromonaco, P.R. 1998. Intimacy as an interpersonal process: the importance of self-disclosure, partner disclosure, and perceived partner responsiveness in interpersonal exchanges. Journal of Personality and Social Psychology, 74, 5, 12381251.

[23] Borchers, T. Self-Disclosure. 1999. Retrieved March 16, 2012 from http://www.abacon.com/commstudies/interpersonal/indisclos ure.html

[24] Siino, R.M., Chung, J. and Hinds, P.J. 2008. Colleague vs. Tool: effects of disclosure in human-robot collaboration. In 17th IEEE International Symposium on Robot and Human Interactive Communication 2008, Münich.

[25] Tapus, A. and Mataric, M.J. 2007. Emulating Empathy in Socially Assistive Robotics. In AAAI Spring Symposium: Multidisciplinary Collaboration for Socially Assistive Robotics 2007, Palo Alto, California.

[26] Davis, M.H. 1983. Measuring individual differences in empathy: evidence for a multidisciplinary approach. Journal of Personality and Social Psychology, 44, 113-126.

[27] Wainer, J., Feil-Seifer, D.J., Shell, D.A. and Mataric, M.J. 2007. Embodiment and Human-Robot Interaction: A TaskBased Perspective. In 16th IEEE International Conference on Robot \& Human Interactive Communication 2007, Jeju, Korea.

[28] Fasola, F. and Mataric, M.J. 2011 Comparing Physical and Virtual Embodiment in a Socially Assistive Robot Exercise Coach for the Elderly. Center for Robotics and Embedded Systems, Los Angeles, CA.

[29] Deshmukh, A., Aylett, R., Kriegel, M. and Vargas, P.A. 2012. Multiple embodiments for robots in heritage applications. In First international conference robotic innovation for cultural heritage 2012, Venice, Italy.

[30] Powers, A., Kiesler, S., Fussell, S. and Torrey, C. 2007. Comparing a Computer Agent with a Humanoid Robot. In HRI 2007, Arlington, Virginia.

[31] Cohen, I., Looije, R., Neerincx, M.A. Child's perception of robot's emotions: effects of platform, context and experience. International Journal of Social Robotics (in press).

[32] Read, J. and MacFarlane, S. 2002. Endurability, Engagement and Expectations: Measuring Children's Fun. In Interaction Design and Children, Vol. 2. Shaker Publishing, Eindhoven. 